\title{
Harmonic quiet-day curves as magnetometer baselines for ionospheric current analyses
}

\author{
M. van de Kamp \\ Finnish Meteorological Institute, P.O. Box 503, 00101 Helsinki, Finland
}

Correspondence to: M. van de Kamp (kampmax@fmi.fi)

Received: 28 February 2013 - Published in Geosci. Instrum. Method. Data Syst. Discuss.: 18 March 2013

Revised: 9 October 2013 - Accepted: 18 October 2013 - Published: 29 November 2013

\begin{abstract}
This paper presents a novel method to determine a baseline for magnetometer data. This baseline consists of all magnetic field components not related to ionospheric and magnetospheric disturbances, i.e. all field components due to solar quiet variations and other background variations, such as tidal and secular variations, as well as equipment effects. Extraction of this baseline is useful when the magnetic field variations due to solar disturbances are analysed. This makes magnetometer data suitable, for instance, for the calculation of ionospheric equivalent currents related to geomagnetic storms and substorms.

The full baseline is largely composed of two main constituents: the diurnal baseline and the long-term baseline. For the diurnal baseline, first "templates" are derived, based on the lowest few harmonics of the daily curves from the quietest days. The diurnal variation of the baseline is obtained by linear interpolation between these templates; this method ensures a smooth baseline at all times, avoiding any discontinuities at transitions between days. The long-term baseline is obtained by linear interpolation between the daily median values of the data; this way the baseline is ensured to follow long-term trends, such as seasonal and tidal variations, as well as equipment drift. The daily median values are calculated for all days except the most disturbed ones; a procedure for this selection is included.

The method avoids many problems associated with traditional baseline methods and some of the other recently published methods, and is simpler in procedure than most other recent ones. As far as can be compared, the distribution of the resulting field after removal of the baseline is largely similar to that using other recent baseline methods. However, the main advantage of the method of this paper over others is that it removes equipment drift and other artefacts efficiently
\end{abstract}

without discarding too much data, so that even low-quality data from remote unmanned magnetometers can be made suitable for analysis. This can give valuable contributions to the database of ionospheric equivalent currents, especially in the area near the polar cap boundary.

\section{Introduction}

\subsection{Equivalent currents}

Currents flowing in the ionosphere are related to the fieldaligned currents in the magnetosphere through ionospheremagnetosphere coupling (e.g. Kamide and Baumjohann, 1993). The magnetospheric currents are strongly dependent on solar activity, such as solar wind variations and solar storms, and these solar-dependent magnetospheric current variations are also reflected to the ionospheric currents. Since strong disturbances in the ionospheric currents can affect technological systems on the ground (e.g. Boteler et al., 1998), there is a great interest in the dynamics of these currents in relation to solar activity.

The concept of ionospheric "equivalent currents" models the ionospheric currents as present in a thin shell, usually the highly conductive E layer at $100 \mathrm{~km}$ height, and representing only the divergence-free, horizontal part of the total currents. Under many circumstances these give valuable information about spatial and temporal characteristics of the actual 3-D ionospheric currents (e.g. Untiedt and Baumjohann, 1993), especially when analysed together with data from other instruments, such as rockets, satellites, or radars. The variations of the equivalent currents can effectively be estimated 
from magnetometer measurements from a two-dimensional ground-based magnetometer network.

The European Cluster Assimilation Technology (ECLAT) (http://www.space.irfu.se/ECLAT/eclat-web/eclatdetail. html) project funded by the EU FP7 programme provides a selection of useful supporting data sets to the Cluster Active Archive (http://caa.estec.esa.int/caa) (Laakso et al., 2009). The work described in this paper has been used in an ECLAT work package in which ionospheric equivalent current vectors caused by solar disturbances are computed in the Fennoscandia region from data from the ground magnetometer network IMAGE. These source data are described in the next subsection. In the project, equivalent currents are to be analysed for an area over Fennoscandia, over the period 2001-2010. An important motivation for publishing this paper is to provide for the ECLAT database users accurate information about the equivalent current generation procedure used in the service.

For the equivalent current estimate, use is made of the method of spherical elementary current systems (Amm and Viljanen, 1999; Pulkkinen et al., 2003). The currents induced inside the earth are ignored, as they can be assumed to be relatively small compared to the ionospheric currents. For the calculation, only the $x$ (north) and $y$ (east) components of the magnetic field at all ground stations are necessary, as only these are related to the divergence-free part of the ionospheric currents.

\subsection{IMAGE magnetometer measurements}

The input data for the calculation of the ionospheric currents in the project ECLAT are the magnetometer recordings of the ground magnetometer network IMAGE (http: //space.fmi.fi/image/beta/) (Viljanen and Häkkinen, 1997). This network consists of 32 magnetometers over geographic latitudes from 58 to $79^{\circ} \mathrm{N}$, which is especially favourable for electrojet studies. The magnetometers return data at a time resolution of $10 \mathrm{~s}$. Figure 1 shows a map of the locations of the magnetometers of the IMAGE network.

\subsection{Various magnetic field components: baselines}

In addition to the currents caused by solar and magnetospheric disturbances (storms and substorms), which are of interest for the analysis, the ionospheric currents and the ground magnetic field consist of several other components.

The magnetic field measured at the earth's surface is a superposition of the field originating from the inner earth, and that from electromagnetic effects caused by the ionospheric currents. These ionospheric currents, in turn, contain on the one hand components due to the dynamo effect of the revolving magnetic earth inside the conducting ionosphere, whose conductivity varies, depending (among others) on solar radiation, and on the other hand components due to storms and substorms caused by coronal mass ejections and other
IMAGE Magnetometer Network

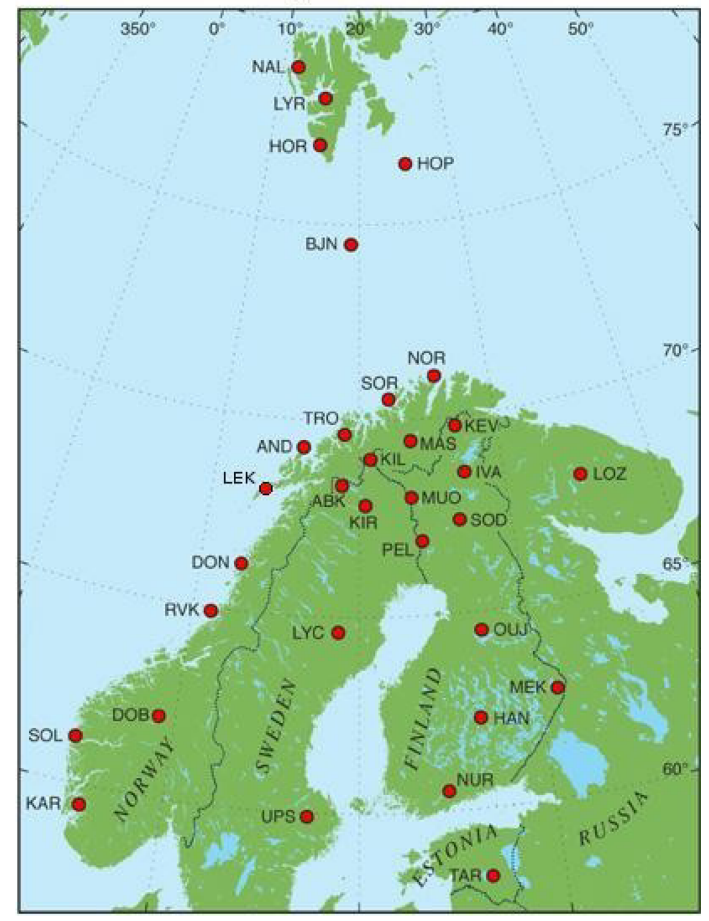

Fig. 1. Map of the IMAGE magnetometer stations.

disturbances in solar activity, and transferred to the ionosphere via ionosphere-magnetosphere coupling. The latter are the components of interest for the ionospheric studies.

As a consequence, in the measured magnetic field the following variations of different timescales can be distinguished:

- the diurnal variations caused by the variation of ionospheric conductivity due to solar radiation during quiet times, usually referred to as " $S_{q}$ variations" or "solar quiet variations". Although this variation is diurnal, its characteristics can change from day to day.

- lunar variations: variations over a lunar day $(24 \mathrm{~h}$ $50 \mathrm{~m}$ ), due to changes in the global distribution of the conducting ionosphere due to lunar attraction. These are usually referred to as " $L$ variations".

- seasonal variations: variations with a period length of one year, due to seasonal changes in the ionospheric conductivity (i.e. slow component of $S_{q}$ variations), as well as seasonal changes in the ionospherethermosphere-magnetosphere interactions.

- secular (long-term) variations, due to very slow variations in the earth's internal magnetic field pattern.

- variations due to solar storms and substorms. These variations are irregular in nature and contain strong fast fluctuations. Still, the nature of these variations 
can show statistical dependencies on time of day, season, and solar activity.

- in addition to the various field variation components, some magnetometer data also contain system effects such as equipment drift (see later).

Note that within the time range of days, lunar, seasonal and secular variations are much smaller than the solar quiet variations and those due to (sub)storms. The various components of the ground magnetic field are clearly explained by Chapman and Bartels (1940). Campbell (1989) gave an introduction on the physics of $S_{q}$ and $L$ variations and a short overview of the research history of these, as an editorial to a special issue of Pure and Applied Geophysics, dedicated to $S_{q}$ and $L$ variations.

In the context where the effects of the solar storms and substorms are assessed and analysed by calculating ionospheric equivalent currents, all magnetic field components unrelated to this should be subtracted from ground-based magnetic field measurements, before further analysis. In this context, the earth's main magnetic field, all the $S_{q}$ and $L$ variations, seasonal variations, and system effects in the output data of any magnetometer are here treated together as "baselines", indicating the components to be removed. The remaining component is assumed to contain only the variations due to solar storms and substorms, and will be referred to in this paper as "disturbance field".

(Note, however, that the term "baseline" has sometimes also been used in the context of studying $S_{q}$ variations, where the term represented the relatively long-term variations. For instance, when Matsushita (1968) referred to the $S_{q}$ study by Price and Wilkins (1963), he used the term "base line" for the variations of the midnight field from day to day.)

Although the magnetometer baseline method of this paper is introduced as the basis of calculating ionospheric equivalent currents, this method can also be useful for any other application where either the disturbance magnetic field or the $S_{q}$ field is to be analysed.

\subsection{Baseline removal}

A traditional method of removing a baseline from magnetometer data of a particular day is to look for a magnetically quiet day near the day of interest, and calculate the average value of the magnetic field of this day. This constant value is used as the baseline, and subtracted from the data for the day of interest, leaving only the disturbance field. For instance, Davis and Sugiura (1966) proposed to derive the auroral electrojet (AE) index from magnetometer data using this as a baseline method.

Although it has long been known that this way the $S_{q}$ variations are not included in the baseline and will therefore be considered as part of the disturbances, it was considered that these variations are small compared to the disturbance field, and hence the introduced error is relatively small.
The magnetically "quiet" days used can be more or less frequent, varying from about one to five quiet days per month. They can be determined in various ways (e.g. based on the variations of the data of each day, or using global magnetic indices as $\mathrm{Kp}$ or Dst). Also, they can be downloaded directly from a global database (http://www-app3. gfz-potsdam.de/kp_index/definitive.html).

The baseline method using averages of quiet days leads to inaccuracies in the resulting disturbed data in several ways:

1. there may not be any day in the entire month which is completely free from disturbances. In this case, the "quiet" day is not really "quiet" and the data, and even the average value, will still be affected by some disturbance effects.

2. as mentioned above, the diurnal variation of the $S_{q}$ field is not included in the baseline and will hence be considered as part of the disturbance field.

3. for two consecutive days, the "nearest" quiet day and therefore the baseline value can be different, and hence the baseline may show a discontinuity at midnight between these two days. If this baseline is subtracted from other data for a period around midnight (e.g. to calculate equivalent ionospheric currents), this may cause an artificial jump in the resulting disturbed data.

4. the magnetometer data generally contain slow variations over the course of several days, months and years, caused by long-term magnetic field variations, as well as equipment effects (see Sect. 2.2 for examples of each). As a consequence, the average value of the quiet day may not be representative of that of other days which can be several weeks earlier or later.

In spite of its drawbacks, the above-mentioned baseline procedure is still being used in some applications today, mainly because of its simplicity. Alternatively, many studies have also been performed using more elaborate baseline procedures. In particular, $S_{q}$ variations (point 2 ) have been incorporated in baselines by appropriate smoothing of the quietday data in one way or the other. Below, a few of the most recent baseline procedures are summarised. It should be emphasised that this is not intended to be a complete review of baseline development, but only to show some examples of the current state of the art.

Janzhura and Troshichev (2008) presented a running automatic method which overcomes some of the problems: point 1 is avoided by looking instead of for quiet days, for any quietest bits of data within a 30-day period; point 2 is avoided by only smoothing the quiet daily curve, but retaining the diurnal variations. Point 4 is only reduced, not entirely removed, by averaging all quiet data over the 30-day period.

However, their method retains point 3 , and even introduces more discontinuities. Since the smoothed daily baseline is in 
general at the end of the day not the same as that at the start of the day, a discontinuity becomes more likely to occur at every midnight. This can be seen, for example, from Fig. 5 in the paper by Janzhura and Troshichev (2008) (the values of the curves at the end of the day do not connect to those at the start of the day).

More recently, Stauning (2011) presented another version of the procedure from Janzhura and Troshichev (2008). The main difference with their method is that his method carefully selects data from similar conditions as the day in question, by giving larger weights to quiet data from days close to the day in question, as well as to data from days approximately 27 days (one solar rotation) before and after. This is because he finds the highest correlation coefficients for such displacement periods. This way, his method further reduces point 4 as long as the slow variations are fluctuations over periods of 27 days. In addition, very slow fluctuations are removed separately in his method. However, his method does not take care of unexpected fluctuations, such as instrument drifts over only a few days (see Sect. 2.2). Also, it is not certain whether any midnight discontinuities (due to point 3, or those as introduced by Janzhura and Troshichev (2008)) remain.

Gjerloev (2012) described the automatic data preprocessing procedure of the worldwide magnetometer network SuperMAG, which also involves a baseline removal technique. Acknowledging the problems associated with the identification of quiet days, he avoids using these altogether, thus avoiding point 1 . Instead, he averages the data from several days around the day of interest ( 3 days in case of relatively quiet periods; longer in disturbed periods), to obtain a daily trend, and applies appropriate smoothing, avoiding point 2 . Point 4 is taken care of by separately determining seasonal variations (referred to as "yearly trend"), although it is not clear how well this works to capture relatively fast and irregular equipment drifts. Also here it is not clear whether the midnight discontinuities remain.

In this context it is good to note that all of the above methods were designed to work for rather well-controlled stable instrumentation, in which case point 4 represents only slow and regular variations, which are well taken care of by these methods.

In this paper, however, a method is described which overcomes all problems in the list above, including in the case of unstable instrumentation, and in addition is simpler than the other methods described above. The procedure will be described in the next section.

\section{Procedure}

The baseline procedure is performed for each magnetometer station separately. The following sections describe the various steps in this procedure.

\subsection{Data jumps}

Many of the magnetometer time series, especially those from remote unmanned stations, exhibit occasional artificial discontinuities, or "jumps", in the data. These can vary in size from a few nanoteslas to thousands of nanoteslas, and can be both positive and negative. The jumps are probably due to adjustments and resetting of the equipment, and happen mostly at midnight, but not only. These jumps, if not taken care of, will affect the results of the data analysis.

Small discontinuities in the time series need not be a problem in a statistical analysis of the magnetic field. However, they do become important when dynamics aspects of geomagnetic events are analysed. For example, the results of the time derivative of the magnetic field will be affected by artificial jumps. Viljanen et al. (2001) analysed the time derivative of the horizontal magnetic field vector from the IMAGE network, and found significant correlations between this parameter and geomagnetic activity, on various timescales from hours up to years, as well as a significant directional variability of this parameter, which in turn also depends on location and time. Plausible relations were found with, among other things, ionospheric currents, pulsations, and geomagnetically induced currents. Because of this, it is evidently important to avoid the measured time derivative being contaminated by artificial discontinuities. (For the same reason, the artificial jumps in magnetometer data introduced by the baselines, i.e. problem 3 in Sect. 1.4, should also be avoided.)

Furthermore, the data jumps will also affect the baseline determination described in this paper, which involves, among other things, some curve fitting and calculation of standard deviations (see later). Because of this, as a first step in the baseline procedure, these jumps need to be removed from the measured data.

The jumps are removed as follows. A software module has been designed which detects and displays suspected discontinuities in the data. The software lets the user examine each discontinuity and decide whether it is artificial, in which case it is listed in a file. Based on this information, a jump baseline $B_{\mathrm{J}}(t)$ is generated, separately for the $x$ and $y$ components of the field, which contains all the jumps found in the data in the form of a superposition of step functions, and is otherwise constant. This jump baseline is subtracted from the raw data, before any further processing as described in the following sections.

\subsection{Long-term baseline}

Next, to determine the baseline, first the part is considered which consists of the long-term variations in the magnetic field (i.e. variations over periods longer than $24 \mathrm{~h}$ ): on the scale of days, months, or years. These variations will be represented in the baseline by determining from every day of data a single daily "background" value. For this value, it might be considered to use the average of the measured data. 

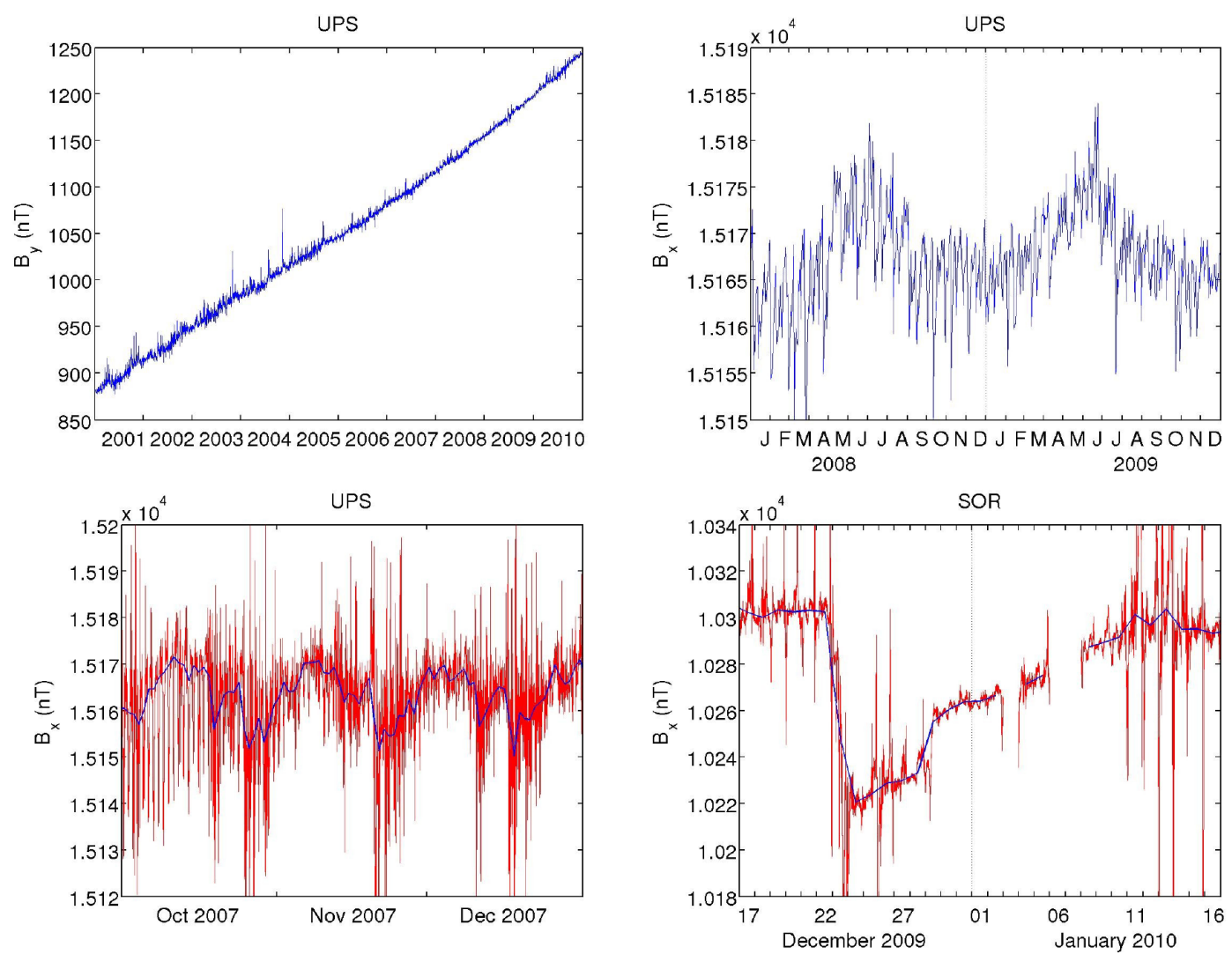

Fig. 2. Daily median values of magnetometer data (blue), demonstrating the long-term variations. Top left panel: secular variation in $B_{y}$ in Uppsala over the full $10 \mathrm{yr}$ period; top right panel: seasonal variations in $B_{x}$ in Uppsala over 2008-2009; bottom left panel: tidal effects in $B_{x}$ in Uppsala in autumn 2007 (raw data included in red); bottom right panel: equipment drift in $B_{x}$ in Sørøya in December 2009 (raw data in red).

However, rather than this, the median is considered more stable, being less sensitive to extreme values during moderately disturbed days.

Figure 2 shows this daily median calculated over various periods of data, and demonstrates the long-term variations revealed by it. This graph shows that these variations consist of, among others, the following components:

- Secular variations: due to very slow variations in the earth's internal magnetic field pattern, the $y$ and $z$ components of the magnetic field at all stations steadily increased, at a rate of slightly over $35 \mathrm{nT} \mathrm{yr}^{-1}$, during the period 2001-2010. (The $x$ component did not change significantly.) The upper left-hand panel of Fig. 2 demonstrates this for $B_{y}$ in Uppsala.

- Seasonal variations: the seasonal changes in the ionospheric conductivity and in the ionospherethermosphere-magnetosphere interactions result in variations in all magnetic field components with a period length of one year. As an example, the upper right-hand panel of Fig. 2 shows that $B_{x}$ in Uppsala oscillated at about $10 \mathrm{nT}$ peak-to-peak within the years of 2008 and 2009.

- Tidal variations: variations with periods of about 27 days can be observed, which are the result of interference between $S_{q}$ and $L$ variations. As an example, the lower left-hand panel of Fig. 2 shows that $B_{x}$ in Uppsala varied at this frequency at about $15 \mathrm{nT}$ peak-to-peak from October to December 2007. (Note that the raw data, which are also shown (red), include the disturbance field and therefore also show statistical variations due to the period of solar activity changes, which also have a period of 27 days. The median values however should not be much affected by this.)

- Equipment drift: some of the magnetometers occasionally exhibit some variations over the course of one or a few days, which do not repeat, and show no correlation with space weather parameters or with any of the other magnetometer results. The lower right-hand panel of Fig. 2 shows an example for Sørøya, where on 23 December 2009 , the readings for $B_{x}$ decreased by $80 \mathrm{nT}$ 
over two days, and then gradually recovered, over the course of 18 days.

The equipment drifts especially happened at the stations in remote locations, which are not continuously manned and monitored. These drifts should obviously be classified as measurement errors. However, if they can be quantified using the current method, they can be removed along with the above-mentioned long-term variations in the magnetic field, which means that these low-quality data need not be discarded. This is a very useful outcome, since these magnetometer stations in remote places often are some of the most crucial ones. A significant number of them are located in northern Scandinavia and the sea between Norway and Svalbard, which is the area above which the auroral oval or its boundaries are often located, and where therefore much of the magnetic activity occurs. But at the same time, not many magnetometers are present there, due to the difficult accessibility of the area. The resulting relevance of the area is precisely why these particular magnetometers were placed in these locations, despite their inaccessibility. It is therefore very useful if these relatively valuable results, even if not of perfect quality, can still be used in ionospheric analyses.

The median is used for the long-term baseline as follows. The median value is calculated for every day of data. The long-term baseline is considered to be equal to the median at 12:00 UT on the respective day. At any other time, the longterm baseline is linearly interpolated between these values. (Note that linear interpolation is good enough, even though it causes "corners" in the data (discontinuities in the first derivative); these corners are not a problem for the magnetic field data as long as the data themselves stay continuous.)

The resulting time-dependent long-term baseline will be referred to in this paper as $B_{\mathrm{T}}(t)$. The procedure to determine $B_{\mathrm{T}}(t)$ is performed separately not only for each different station but also for each field component.

Obviously, during magnetically very disturbed periods, the median value calculated over a day may not be representative of the long-term baseline. In these cases, the median of these particular days will not be used, but the long-term baseline will be interpolated between other median values. Further on, it will be shown how the classification of these "usable" median values is performed.

\subsection{Quiet days}

For every separate magnetometer station, a list of the quietest days of each month is generated.

At any magnetometer station, a day is considered "quiet" if the magnetic field variations measured on this day are (almost) entirely caused by $S_{q}$ variations and not by magnetic disturbances driven by solar activity. Hence, a "quiet" day would mainly contain slow variations; its fast variations should be relatively small.

Because the fast variations due to disturbances are mostly much larger than the $S_{q}$ variations, it seems logical to calculate simply the standard deviation of every day of data, and look for the smallest standard deviations of each month. Indeed this method works reasonably. However, there are cases where this method is too coarse, as will become clear in the description below of the method of this paper.

In this paper, the quiet-day selection is performed as follows. Each day of data is partitioned into 24 one-hour sections (UT). In each one-hour section, a straight line is fitted to the data of the $x$ and $y$ components of the magnetic field. This straight line is subtracted from the data, and from the remaining data, the hourly standard deviation, $\sigma_{\mathrm{H}}$, is calculated. The result of this is $2 \times 24$ values ( 2 components and $24 \mathrm{~h}$ ) of $\sigma_{\mathrm{H}}$. Of these 48 values, the daily maximum is calculated, referred to as $\sigma_{\mathrm{H}_{\max }}$, for each day and each station. These values of $\sigma_{\mathrm{H}_{\max }}$ are the indicators for days with and without disturbances: of each month, the day with the lowest $\sigma_{\mathrm{H}_{\max }}$ is selected as the "quiet day" for that month, at that station.

As an extra requirement, the "quiet day" should contain no data gaps, so only days with $100 \%$ data availability for both magnetic components can be potential candidates for any month's "quiet day".

Figure 3 demonstrates this procedure, showing $B_{x}$ in Oulujärvi on three different days in March 2002. The day shown in the bottom two panels, 28 March, resulted from this procedure as the quietest day of this month (note that the actual procedure also takes into account the $y$ component of the magnetic field, which is not shown in the figure).

Figure 4 demonstrates why this procedure works better than calculating the overall standard deviation of the whole day. On 28 March (the quiet day at the bottom of Fig. 3) the full-day standard deviation of $B_{x}$ is $19.9 \mathrm{nT}$, while on 7 March (Fig. 4), it is smaller: $10.0 \mathrm{nT}$. However, the latter day contains some fast variations, which make it less suitable as a "quiet" day. This property is revealed by the maximum hourly standard deviation $\sigma_{\mathrm{H}_{\max }}$, which is larger on 7 March: $6.7 \mathrm{nT}$ rather than $1.4 \mathrm{nT}$.

Of course, even though according to this procedure a "quietest" day of each month can always be found (as long as at least one full day of data is available), it may be that this quietest day still contains too much magnetic disturbance to be used for a baseline. Especially during and around the solar active year of 2003, disturbances can be so frequent that not necessarily within every month a full day can be found where these disturbances are insignificantly small. In other words: the "quietest" day of the month may not be really "quiet".

Because of this, an extra criterion is applied: if for any month, the value of $\sigma_{\mathrm{H}_{\max }}$ of the quiet day is above a certain threshold value, then this quiet day is discarded and no quiet day is assigned for this month. Later it will become clear that these cases do not cause a problem in the rest of the procedure. The optimum threshold value of $\sigma_{\mathrm{H}_{\max }}$ to be used for this depends on the typical level of both slow and fast variations in the magnetic field. The threshold value was empirically chosen by visual inspection of many data sets from 

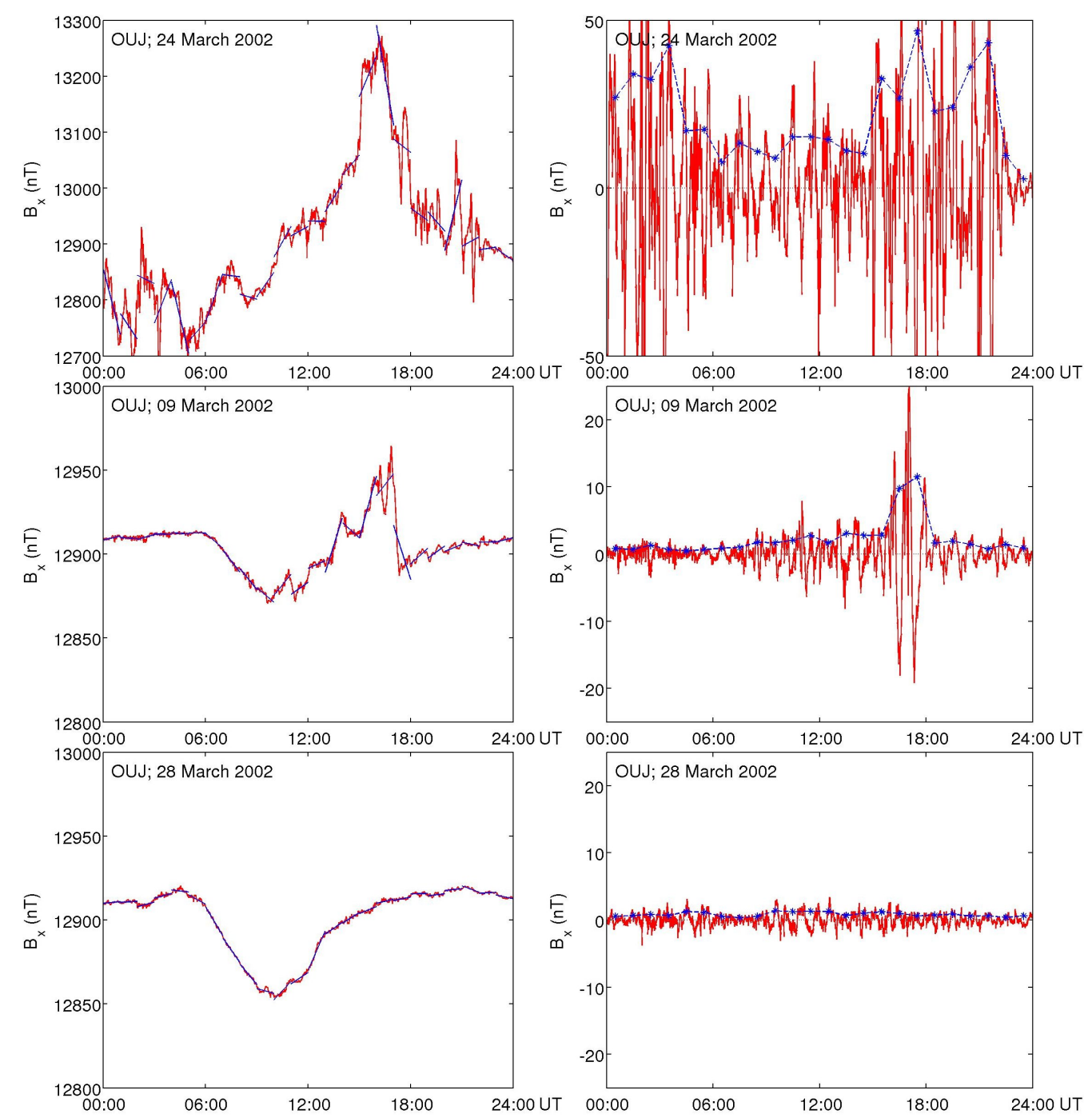

Fig. 3. The quiet-day selection procedure, demonstrated by the $B_{x}$ field in Oulujärvi in March 2002. Left-hand panels: raw data and the hourly lines fitted to it. Right-hand panels: the data after subtraction of the fitted lines, and the calculated hourly standard deviations. Top panels: on a disturbed day; centre panels: on a mostly quiet day with some disturbance; bottom panels: on a quiet day. The maximum $\sigma_{\mathrm{H}}$ values (from $B_{x}$ alone) are 46.711 .5 , and $1.4 \mathrm{nT}$, respectively.
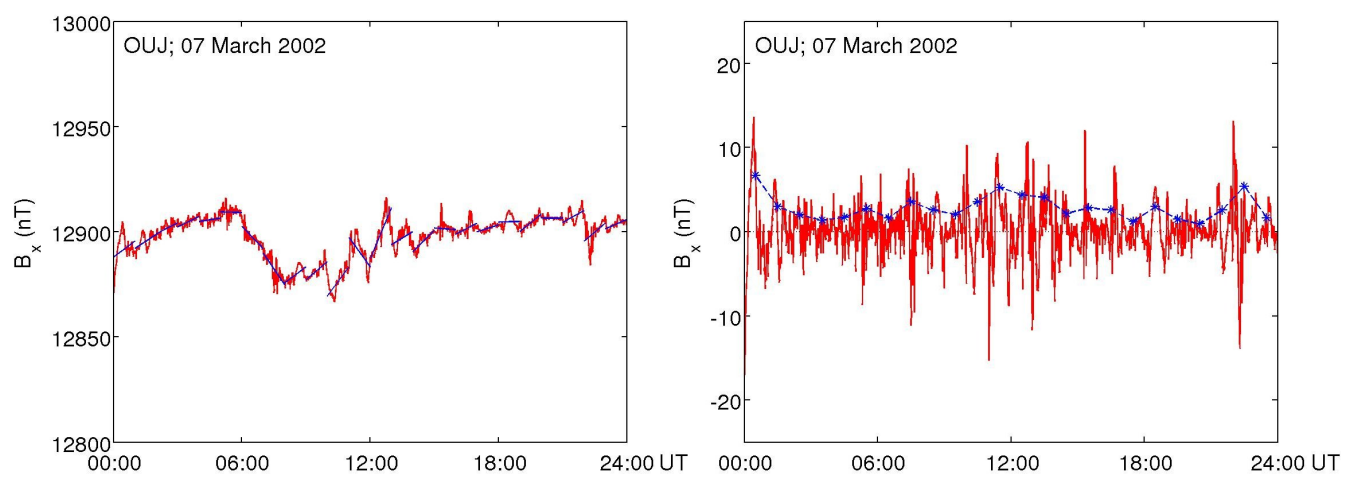

Fig. 4. Similar panels as in Fig. 3, for 7 March 2002, which, although the overall variation is quite small, contains some disturbances. The maximum value of $\sigma_{\mathrm{H}}\left(\right.$ from $B_{x}$ ) is $6.7 \mathrm{nT}$. 
Table 1. Threshold values of $\sigma_{\mathrm{H}_{\max }}$ and $\sigma_{\mathrm{H}_{\text {med }}}$ for each IMAGE station.

\begin{tabular}{|c|c|c|c|c|c|c|}
\hline Station & Name & $\begin{array}{l}\text { Latitude } \\
\quad\left({ }^{\circ}\right)\end{array}$ & $\begin{array}{r}\text { Longitude } \\
\left({ }^{\circ}\right)\end{array}$ & $\begin{array}{r}\text { Threshold } \\
\sigma_{\mathrm{H}_{\max }}(\mathrm{nT})\end{array}$ & $\begin{array}{r}\text { Threshold } \\
\sigma_{\mathrm{H}_{\text {med }}, B_{x}(\mathrm{nT})}\end{array}$ & $\begin{array}{r}\text { Threshold } \\
\sigma_{\mathrm{H}_{\mathrm{med}}}, B_{y}(\mathrm{nT})\end{array}$ \\
\hline NAL & Ny-Ålesund & 78.92 & 11.95 & 18 & 14 & 13 \\
\hline LYR & Longyearbyen & 78.20 & 15.82 & 21 & 16 & 15 \\
\hline HOR & Hornsund & 77.00 & 15.60 & 25 & 20 & 17 \\
\hline HOP & Hopen Island & 76.51 & 25.01 & 29 & 22 & 14 \\
\hline BJN & Bjørnøya & 74.50 & 19.20 & 28 & 21 & 15 \\
\hline NOR & Nordkapp & 71.09 & 25.79 & 19 & 25 & 12 \\
\hline SOR & Sørøya & 70.54 & 22.22 & 20 & 20 & 12 \\
\hline KEV & Kevo & 69.76 & 27.01 & 14 & 18 & 9 \\
\hline TRO & Troms $\varnothing$ & 69.66 & 18.94 & 17 & 20 & 11 \\
\hline MAS & Masi & 69.46 & 23.70 & 13 & 18 & 11 \\
\hline AND & Andenes & 69.30 & 16.03 & 17 & 20 & 11 \\
\hline KIL & Kilpisjärvi & 69.06 & 20.77 & 13 & 18 & 10 \\
\hline IVA & Ivalo & 68.56 & 27.29 & 10 & 16 & 8 \\
\hline $\mathrm{ABK}$ & Abisko & 68.35 & 18.82 & 11 & 17 & 10 \\
\hline LEK & Leknes & 68.13 & 13.54 & 10 & 15 & 9 \\
\hline MUO & Muonio & 68.02 & 23.53 & 9 & 15 & 8 \\
\hline $\mathrm{LOZ}$ & Lovozero & 67.97 & 35.08 & 8 & 12 & 6 \\
\hline KIR & Kiruna & 67.84 & 20.42 & 9 & 14 & 8 \\
\hline SOD & Sodankylä & 67.37 & 26.63 & 8 & 13 & 7 \\
\hline PEL & Pello & 66.90 & 24.08 & 7 & 12 & 7 \\
\hline DON & Dønna & 66.11 & 12.50 & 7 & 11 & 6 \\
\hline RVK & Rørvik & 64.94 & 10.98 & 6 & 9 & 5 \\
\hline LYC & Lycksele & 64.61 & 18.75 & 8 & 9 & 7 \\
\hline OUJ & Oulujärvi & 64.52 & 27.23 & 6 & 7 & 5 \\
\hline MEK & Mekrijärvi & 62.77 & 30.97 & 5 & 4 & 4 \\
\hline HAN & Hankasalmi & 62.25 & 26.60 & 5 & 4 & 4 \\
\hline DOB & Dombås & 62.07 & 9.11 & 4 & 5 & 4 \\
\hline SOL & Solund & 61.08 & 4.84 & 4 & 4 & 4 \\
\hline NUR & Nurmijärvi & 60.50 & 24.65 & 4 & 5 & 3 \\
\hline UPS & Uppsala & 59.90 & 17.35 & 4 & 4 & 3 \\
\hline KAR & Karmøy & 59.21 & 5.24 & 4 & 3 & 3 \\
\hline TAR & Tartu & 58.26 & 26.46 & 4 & 3 & 3 \\
\hline
\end{tabular}

the station Abisko, which shows a continuous set of goodquality data. The optimum value (for this station) was found to be $11 \mathrm{nT}$. This value for $\sigma_{\mathrm{H}_{\max }}$, which coincides with daily median values of $\mathrm{Kp}$ of around 3, is in Abisko exceeded for $8 \%$ of all quiet days, and for a maximum of 3 consecutive monthly quiet days (this is in the particularly active time of April-June 2003).

However, because the optimum threshold value of $\sigma_{\mathrm{H}_{\max }}$ depends on the typical level of both slow and fast variations in the magnetic field, it varies from station to station. The threshold values to be used for all other stations were empirically adjusted, to make sure all different threshold values represent the same level of irregularities in the respective data sets. To do this, for each station, all values of $\sigma_{\mathrm{H}_{\max }}$ were selected which coincided with $\sigma_{\mathrm{H}_{\max }}$ in Abisko being within $11 \pm 1 \mathrm{nT}$, and the median of these values was taken as the threshold for that station. The resulting values are listed for all IMAGE stations in Table 1 (5th column). Using these threshold values, generally no more than three consecutive months without quiet days were encountered for any station in the entire IMAGE database for 2001-2010.

\subsection{Very disturbed days}

The information of the hourly standard deviations $\sigma_{\mathrm{H}}$, calculated as described in the previous section, will also be used to classify certain days as being too disturbed for calculation of the median value, used in the derivation of the long-term baseline $B_{\mathrm{T}}(t)$ (see Sect. 2.2).

In the top panel of Fig. 5, three days of $B_{x}$ data from Abisko in November 2001 are shown, along with their daily median values (green stars). On 4 November, conditions are mostly quiet, and the median value is a good representative of the long-term baseline. On 5 November, some disturbances start late in the day, but the median value is not significantly affected by them. However, on 6 November, conditions are disturbed all day, and the median value is dominated by these 


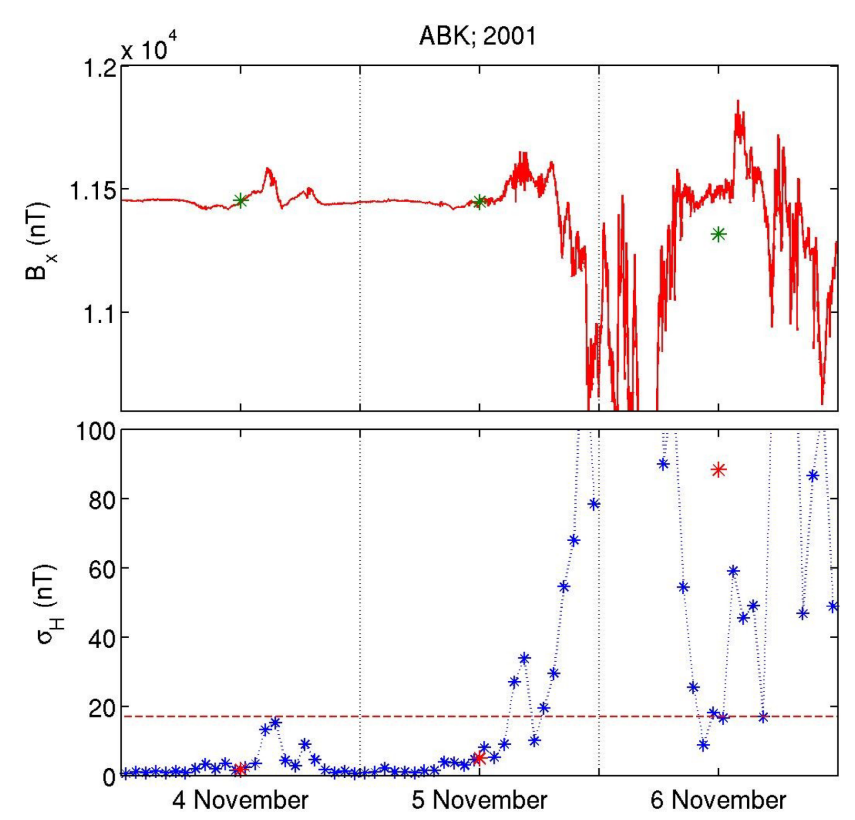

Fig. 5. Top panel: $B_{x}$ in Abisko on 4-6 November 2001, and the daily median values calculated from it (green stars). Bottom panel: hourly standard deviations $\sigma_{\mathrm{H}}$ of these data (blue stars), daily medians $\sigma_{\mathrm{H}_{\text {med }}}$ of these (red stars), and suggested threshold value for $\sigma_{\mathrm{H}_{\text {med }}}$ of $B_{x}$ in Abisko (red dashed line).

disturbances, and unsuitable to be used for the long-term baseline.

A rule of thumb can be described as follows: a median value is relatively insensitive to irregularities in data as long as these irregularities consist of less than half of the data. This can be seen on 5 November in Fig. 5: since the irregularities consist of less than half the day, the median is not significantly affected by them.

Because of this rule, the distribution of hourly standard deviations $\sigma_{\mathrm{H}}$ can serve as a useful indicator for the stability of the daily median value. If more than half of the day's $\sigma_{\mathrm{H}}$ values indicate disturbed data during their hours (as in the previous section), it can be assumed that more than half of the data of the day are disturbed, and the daily median value will be unreliable. On the other hand, if more than half of the $\sigma_{\mathrm{H}}$ values are low, then more than half of the day's data will be quiet, and the median value will be relatively unaffected by any disturbances. These different cases are clearly illustrated in the bottom panel of Fig. 5, where the $\sigma_{\mathrm{H}}$ values of the data in the upper panel are shown (blue stars).

This criterion is easily represented by the median value of the day's hourly standard deviation, which will be referred to as $\sigma_{\mathrm{H}_{\text {med }}}$. If $\sigma_{\mathrm{H}_{\text {med }}}$ is above a certain threshold, this means that at least half the $\sigma_{\mathrm{H}}$ values are above this threshold. This can be seen in the bottom panel of Fig. 5, where $\sigma_{\mathrm{H}_{\text {med }}}$ values are the red stars. As a threshold value of $B_{x}$ for Abisko, $17 \mathrm{nT}$ was empirically chosen by visual inspection of many data series. This value, which coincides with daily median values

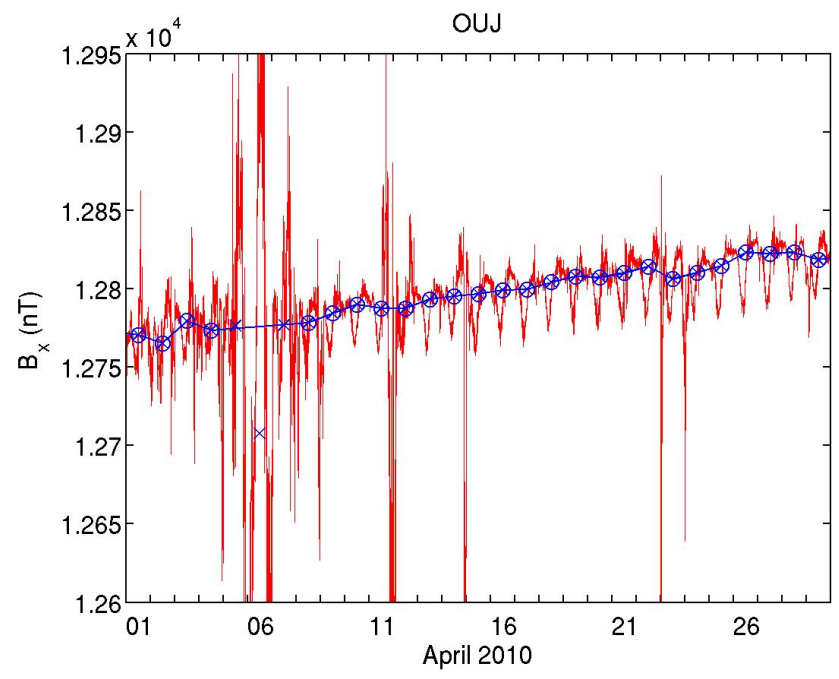

Fig. 6. $B_{x}$ field in Oulujärvi in April 2010 (red), the daily median values (blue $\mathrm{x}$ ), the ones that are qualified to be used for the longterm baseline (circles), and this baseline $B_{\mathrm{T}}(t)$ (blue solid line).

of $\mathrm{Kp}$ of about 10, is exceeded for $11 \%$ of all days, and for a maximum of 10 consecutive days (in May 2003). The value of $17 \mathrm{nT}$ is indicated as a dashed line in the bottom panel of Fig. 5.

The decision of whether a median value is used for the long-term baseline $B_{\mathrm{T}}(t)$ is made separately for $B_{x}$ and $B_{y}$. Because typical variations in $B_{y}$ are often different from those in $B_{x}$, also different threshold values for these two must be used. Furthermore, the threshold values are dependent on station location, just as is the case for the threshold of $\sigma_{\mathrm{H}_{\max }}$ (see previous section). Because of this, the thresholds for $B_{y}$ in Abisko and for both $B_{x}$ and $B_{y}$ for all other stations were all empirically adjusted in the same way as $\sigma_{\mathrm{H}_{\max }}$ in Sect. 2.3: each time, the median of all values of $\sigma_{\mathrm{H}_{\text {med }}}$ coinciding with $\sigma_{\mathrm{H}_{\text {med }}}$ for $B_{x}$ in Abisko being within $17 \pm 1 \mathrm{nT}$ was taken as the threshold for that component and that station. The resulting values are included in Table 1 (rightmost two columns).

Figure 6 shows an example of the result of this procedure, by the $B_{x}$ field in Oulujärvi in April 2010. These data contain some long-term baseline variations, as well as some irregularities. Using the procedure described in this section, the median values of the entire month except 5-7 April were considered suitable for the long-term baseline. The long-term baseline $B_{\mathrm{T}}(t)$, interpolated between the suitable median values, follows the long-term behaviour well, and is unaffected by the disturbance on 5-7 April.

It may be considered in which cases this procedure might give a wrong result: if, in the case of a solar storm which lasts several days, there is a day with a significant magnetic field deviation due to the storm but very few irregularities, $\sigma_{\mathrm{H}_{\text {med }}}$ might be below the threshold, so that the daily median value, while still affected by the storm, is used for the long-term baseline. In this case this "quiet" part of the storm will be 
Table 2. Frequencies of the 7 lowest harmonics of data of 1 day.

\begin{tabular}{ccl}
\hline$h$ & $f(\mathrm{~Hz})$ & period \\
\hline 0 & 0 & inf \\
1 & $1.1574 \times 10^{-5}$ & 1 day \\
2 & $2.3148 \times 10^{-5}$ & $12 \mathrm{~h}$ \\
3 & $3.4722 \times 10^{-5}$ & $8 \mathrm{~h}$ \\
4 & $4.6296 \times 10^{-5}$ & $6 \mathrm{~h}$ \\
5 & $5.7870 \times 10^{-5}$ & $4 \mathrm{~h} 48 \mathrm{~m}$ \\
6 & $6.9444 \times 10^{-5}$ & $4 \mathrm{~h}$ \\
\hline
\end{tabular}

included in the baseline variation, and removed from the data as if it were a slow $S_{q}$ variation. However, these cases will not be frequent, give only a small contribution to the magnetic field, and, most importantly - they are very hard to tell apart from real $S_{q}$ variations, which should be removed from the data. Because of this, it is considered that the procedure described above works well enough, if no other information is used to tell quiet storm parts apart from $S_{q}$ variations.

\subsection{Templates composed of harmonics}

Coming back to the subject of quiet days, defined in Sect. 2.3, this section describes how the diurnal variations are derived from these quiet days.

The diurnal $S_{q}$ variations of the magnetic field can be approximated by harmonic components, as various researchers have already done in the past. Overviews of results of such harmonic analyses performed since 1889 are given by Matsushita (1968) and Campbell (1989). More recently, another harmonic analysis of $S_{q}$ currents was performed by Pedatella et al. (2011). Following similar principles, in this section the diurnal baseline of the two horizontal components of the ground-measured magnetic field is derived by harmonic analysis of the measured data from quiet days, separately for the $x$ and $y$ components.

For all of the quiet days, and each component, the longterm baseline $B_{\mathrm{T}}(t)$ is subtracted from the data of the entire day. On the residual data, a fast Fourier transform (FFT) is performed. From the result of this, only the lowest few frequency components are used. The exact number of frequency components to be used is somewhat arbitrary; in the method of this paper up to the 6th component is used, similarly as in the $S_{q}$ study by Pedatella et al. (2011), and in the determination of the Dst index by Sugiura (1964). This means that only the first 7 (the 0th through the 6th) values resulting from the FFT are used. These complex values represent the amplitudes and phases of harmonics with frequencies which are all multiples of the inverse of 1 day. The frequencies of the first 7 harmonics are given in Table 2. Effectively, this means that $S_{q}$ variations are assumed to be confined to periods of $4 \mathrm{~h}$ and longer, while the disturbance field will contain all faster components.

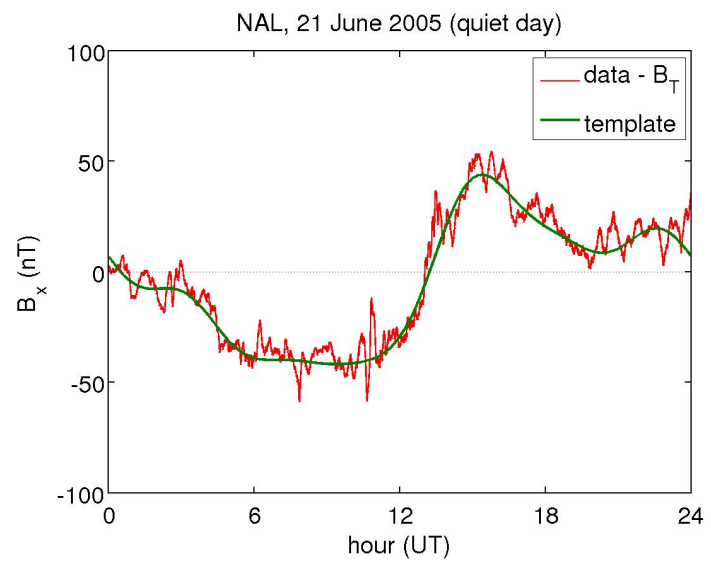

Fig. 7. Red: $B_{x}$ field measured by the magnetometer in Ny-Ålesund (NAL) on 21 June 2005, one of the assigned quiet days, with the long-term baseline subtracted, leaving only the diurnal variation. Green: the template derived from this quiet day.

The curve, composed of these 7 lowest harmonics of the quiet day, is equivalent to a low-pass-filtered version of the quiet-day data. The resulting curve, which will here be called a "template", will be used as a basis of the baseline construction. The template is described as follows:

$T\left(t_{\mathrm{d}}\right)=\sum_{h=0}^{6}\left|X_{\mathrm{h}}\right| \cos \left(\frac{2 \pi h t_{\mathrm{d}}}{86400}+\arg \left(X_{\mathrm{h}}\right)\right)$,

where $t_{\mathrm{d}}=$ "time of day", time elapsed since midnight (s); $h=$ index number of harmonic $(0 \ldots 6)$, and $X_{\mathrm{h}}=$ (complex) coefficient of harmonic $h(\mathrm{nT})$.

There will be one set of harmonic coefficients $X_{\mathrm{h}}$, and therefore one template $T(t)$, defined for each quiet day (and each field component). One example day of data, with the long-term baseline $B_{\mathrm{T}}(t)$ subtracted, as well as the template derived from this, is shown in Fig. 7.

It is worth noting that since all the cosine arguments in Eq. (1) cover a whole number of cycles over the length of one day $(86400 \mathrm{~s})$, the template value at midnight at the end of the day (i.e. $T(86400 \mathrm{~s})$ ) will always be equal to that at midnight at the start $(T(0 \mathrm{~s}))$, thus ensuring continuity at midnight if the template were to be used on consecutive days. However, the templates are not used directly as such for the baselines, which will be shown in the next subsection.

\subsection{Diurnal baseline}

As the next step, a curve representing the diurnal $S_{q}$ variations (i.e. the diurnal variation of the background magnetic field) is derived from the templates, separately for the $x$ and $y$ components. This curve will be referred to as the "diurnal baseline", and will be expressed as $B_{\mathrm{S}}(t)$.

To obtain the diurnal baseline, the templates are linearly interpolated continuously between midday on the previous 


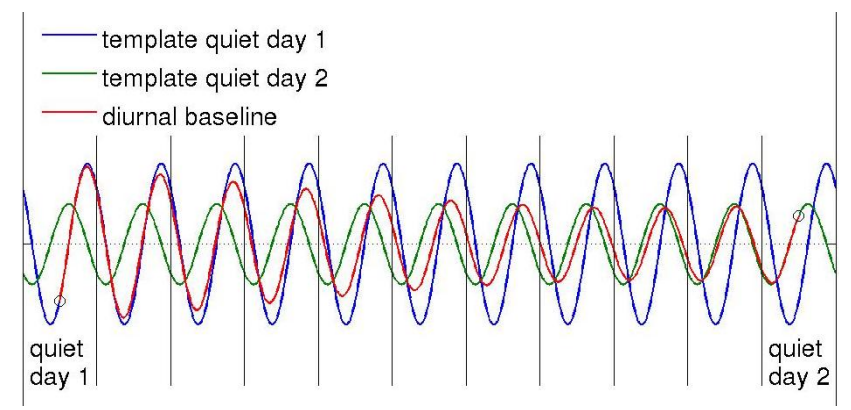

Fig. 8. The principle of interpolation between templates. Templates derived from two consecutive quiet days, and the diurnal baseline, linearly interpolated between them. The vertical lines mark midnights (separation between days).

assigned quiet day and midday on the next assigned quiet day. This can be expressed as follows:

$B_{\mathrm{S}}(t)=T_{1}\left(t_{\mathrm{d}}\right)+\left(T_{2}\left(t_{\mathrm{d}}\right)-T_{1}\left(t_{\mathrm{d}}\right)\right) \frac{t-t_{1}}{t_{2}-t_{1}}$,

where $t=$ the time point of interest (s), $t_{\mathrm{d}}=$ "time of day" as in Eq. (1), $T_{1,2}=$ the template as a function of time of day on the previous and next quiet day, respectively, and $t_{1,2}=$ the time point of midday on the previous and next quiet day, respectively (s).

It should be noted that, for the sake of consistency, also on the quiet days themselves the templates are interpolated. Consequently, only at noon, the diurnal baseline of a quiet day is exactly equal to the template of the same day. After noon, it is linearly interpolated between this template and the next template, and before noon, it is interpolated with the previous template.

Figure 8 shows a schematic example, using imaginary templates consisting of only 1 st harmonics. The blue curve is the template derived from quiet day 1 (the day on the left side); the green curve is the template from quiet day 2 (on the right side). The diurnal baseline (red curve) is linearly interpolated between these two from midday on the first quiet day to midday on the second quiet day. Only at noon on the quiet days (marked as "o" in the graph), the baseline is exactly equal to a template.

Note that the cases of months without any quiet day, which can occur during some of the most disturbed periods in solar maximum years (see Sect. 2.3), do not cause a serious problem in this procedure; they merely mean a longer period between two quiet days over which the template is interpolated. To give an indication of how long these periods can be: in the current database, the largest amount of data between any two quiet days was 66 days of data, over a period of 99 days (Masi station; between 6 February and 17 May 2003).

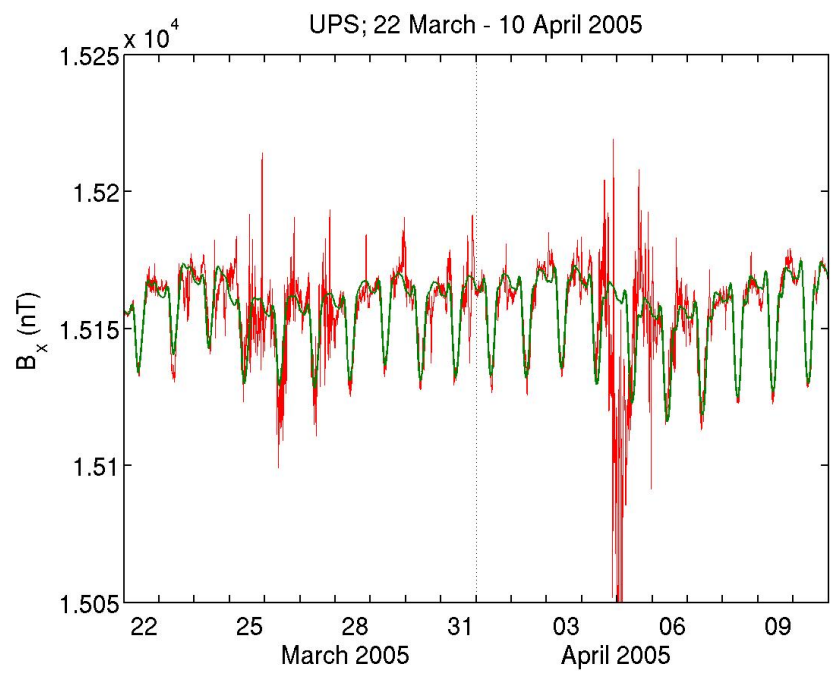

Fig. 9. $B_{x}$ field at Uppsala from 22 March to 10 April 2005 (red), and the corresponding baseline derived as described in this paper (green).

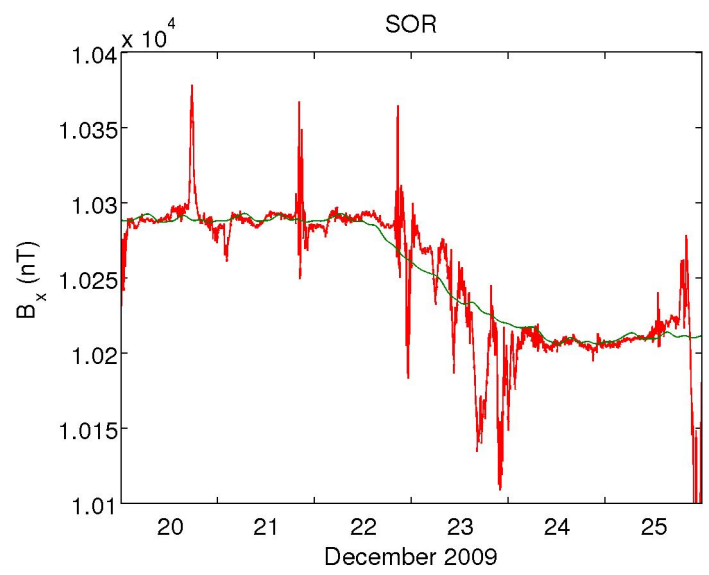

Fig. 10. $B_{x}$ field at Sørøya from 20 to 25 December 2009, and the corresponding baseline derived as described in this paper.

\subsection{Full baseline}

To obtain the full baseline, separately for the $x$ and $y$ components, the diurnal baseline $B_{\mathrm{S}}$ is added to the long-term baseline $B_{\mathrm{T}}$ and the jump baseline $B_{\mathrm{J}}$ :

$B_{\mathrm{B}}(t)=B_{\mathrm{S}}(t)+B_{\mathrm{T}}(t)+B_{\mathrm{J}}(t)$,

where $B_{\mathrm{B}}=$ full baseline; $B_{\mathrm{S}}=$ diurnal baseline, derived from quiet days as described in Sects. 2.3, 2.5 and 2.6; $B_{\mathrm{T}}=$ longterm baseline, derived from suitable median values as described in Sects. 2.2 and 2.4; and $B_{\mathrm{J}}=$ jump baseline, containing only the data jumps as described in Sect. 2.1.

Figure 9 presents an example of the result of the procedure described in this section. It shows the $B_{x}$ field in Uppsala from 22 March to 10 April in 2005 (two quiet days), and the corresponding baseline. In this example, the diurnal 


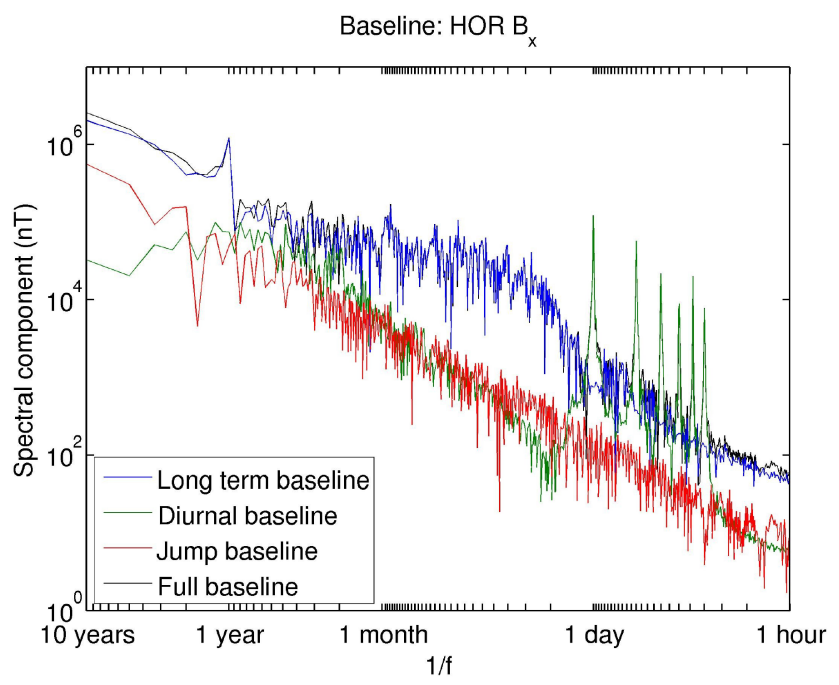

Fig. 11. Spectra of the different baseline components, of the $B_{x}$ field in Hornsund: blue: long-term baseline; green: diurnal baseline; red: jump baseline; black: full baseline. The $x$ axis labels show the inverse of the frequency $f$.

variation of the baseline follows that of the data not only on the assigned quiet days but also on relatively quiet intervals in the middle of this period (e.g. 1 April). Furthermore, the long-term (mostly tidal) variation of the data is well followed by the baseline.

Figure 10 shows an example for the $B_{x}$ field in December 2009 in Sørøya, which experienced some significant equipment drift in this period (also shown in Fig. 2). The figure shows that the baseline derived for this period follows the equipment drift well, making this data reasonably usable for equivalent current calculations.

\section{General results}

The usefulness of the baseline procedure described in this paper is mainly demonstrated by the fact that it avoids all the problems which some other baseline procedures have (as listed in Sect. 1.4), and so makes the resulting data suitable for the calculation of ionospheric equivalent currents. However, it can also be demonstrated by showing some general characteristics of the baselines and the resulting magnetic disturbance field data.

\subsection{Spectral analysis}

As explained in Sect. 2.5, the baseline of this paper is designed to remove only components of periods of $4 \mathrm{~h}$ or longer. This means effectively that the $S_{q}$ variations are assumed to be confined to those periods, while the disturbance field retains all faster components (but not necessarily only those). The effect of this should be reflected in the signal spectrum of each component.

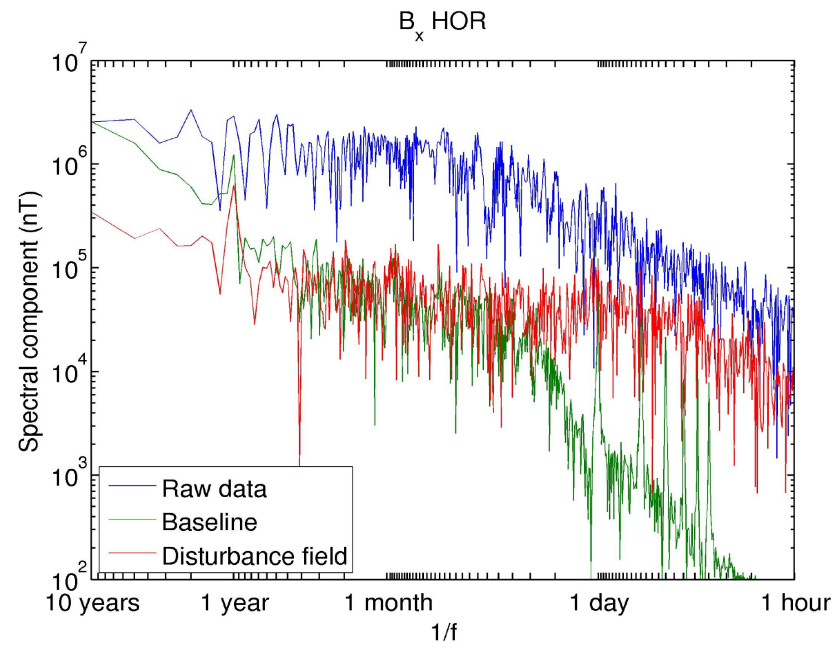

Fig. 12. Spectra of the the $B_{x}$ field in Hornsund (blue), the full baseline (green), and the magnetic field data after removal of the baseline (i.e. the disturbance field; red).

In this section, the different components of the baseline (long-term, diurnal and jump baseline), as well as the full baseline, and the magnetic field measurements are spectrally analysed. This analysis reveals which frequencies are contained in which baseline components and which frequencies remain present in the magnetic field data after removal of the baselines. For this purpose, the $10 \mathrm{yr}$ data (2001-2010) of all stations were read with a time resolution of $30 \mathrm{~min}$, which allows a spectral analysis of frequencies from $1 /(10 \mathrm{yr})$ up to $1 /(1 \mathrm{~h})$.

Figure 11 shows the spectrum for the three baseline components for the $B_{x}$ field in Hornsund. This case is a good representation of the general result of the spectral analysis for the different magnetometers. The graph shows that the long-term baseline (blue) has its strongest frequency contributions in the low-frequency area (periods of months/years). The diurnal baseline (green) generally has a lower spectrum than the long-term baseline, except, as expected, for 6 strong peaks at the frequencies of the harmonic components which make up the template (e.g. see Table 2). The spectrum of the jump baseline (red) is also lower than of the long-term baseline. The spectrum of the full baseline (black) is hard to see in the graph, being mostly hidden behind whichever component has the highest spectrum. Also this decreases with increasing frequency, becoming particularly low for frequencies above the inverse of $4 \mathrm{~h}$.

Figure 12 shows the spectrum of the (full) baseline again, together with those of the raw measured $B_{x}$ field and the field remaining after removal of the baseline (i.e. the disturbance magnetic field). This graph shows that the raw data have a flat spectrum up to about the inverse of half a month, after which it decreases with frequency. Because the baseline contains mainly low-frequency components, mainly those components are removed from the data. As a result, up to 


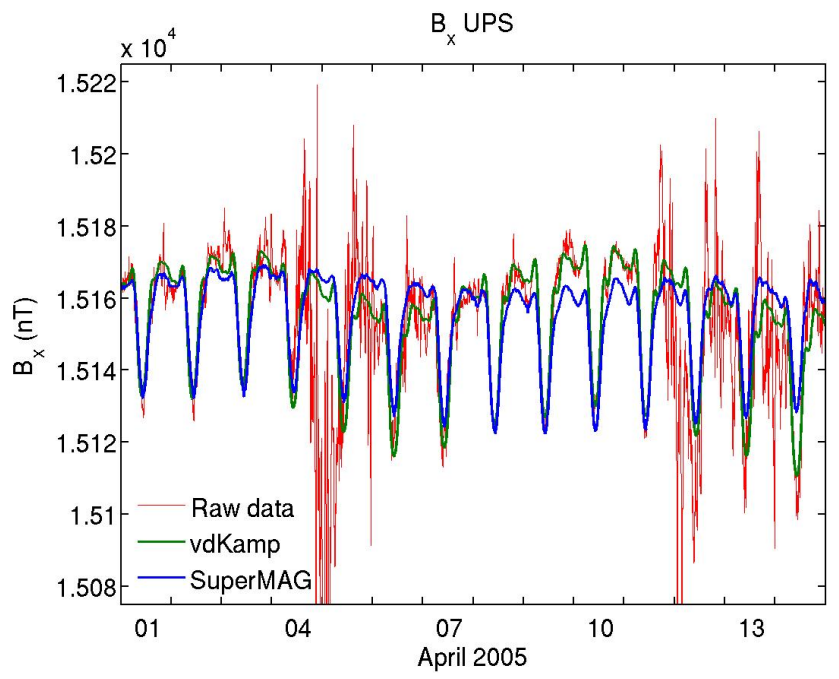

Fig. 13. $B_{x}$ field at Uppsala from 1 to 15 April 2005 (red), the baseline derived as described in this paper (green), and the SuperMAG baseline (blue).

$f=1 /(4 \mathrm{~h})$ the disturbance data have a fairly flat spectrum, resembling that of white noise, which indicates that in that frequency range all regular components have been removed from the data. The disturbance field gets closest to the original data for the highest frequencies.

\subsection{Comparison to other methods}

It is useful to compare the baseline method of this paper to other baseline methods, such as the ones described in Sect. 1.4: those by Janzhura and Troshichev (2008), Stauning (2011), and/or Gjerloev (2012). Unfortunately, the full procedure of those methods is not available to the author of this paper. However, the baseline method by Gjerloev (2012) was used for the data in the SuperMAG database; see http://supermag.jhuapl.edu/mag/ or http: //supermag.uib.no/mag/. This database contains data from magnetometers distributed all over the globe. The baseline used there (hereafter referred to as "SuperMAG baseline") has been retrieved from data downloaded from one of these websites. A comparison with that baseline is presented in this section.

Firstly, a comparison is made of the $B_{x}$ baseline at Uppsala over the entire year of 2005. This year was about midway between the solar maximum and minimum, and shows therefore moderate magnetic disturbances. The raw data in Uppsala are of good quality: no equipment effects can be noted in the data for the entire year 2005. Figure 13 shows the SuperMAG baseline in April 2005 (blue), compared to the the baseline from this paper (green, marked as "vdKamp"), and the raw data (red). (These data are partly the same as the ones shown in Fig. 9.)

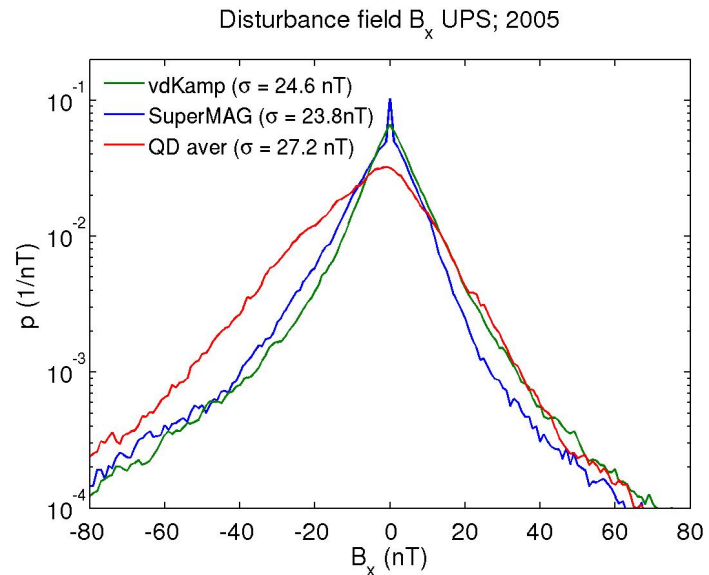

Fig. 14. Probability density function of the $B_{x}$ disturbance field at Uppsala over the year 2005: using the baseline from this paper (green), using the SuperMAG baseline (blue), and using as a baseline the average values of quiet days (red).

This figure shows that the baseline from this paper follows the day-to-day variations more than the SuperMAG baseline (e.g. see on 9-11 April). Variations on this timescale are mostly tidal (i.e. the result of interference between $S_{q}$ and $L$ variations (see Sect. 2.2)). This suggests that some of these tidal variations are likely to remain in the field after removal of the SuperMAG baseline. On the other hand, as explained in Sect. 2.4, it might be that some quiet parts of solar storms are mistaken for tidal variations and included in the baseline of this paper; this could be the case in Fig. 13 on 6-7 April. However, since those quiet parts of solar storms are hard to tell apart from real tidal $S_{q}$ variations, it is not evident from this observation which baseline is better in this case.

Some statistics of these two baselines over 2005 has been calculated: the standard deviation of the baseline of this paper is equal to $13.1 \mathrm{nT}$, while that of the SuperMAG baseline is $10.3 \mathrm{nT}$. This difference is a consequence of the fact that also over the entire year, the baseline according to this paper follows the variations of the raw data more than the SuperMAG baseline, as seen in Fig. 13. Still, the difference between the standard deviations is small. Furthermore, the standard deviation of the difference between the two baselines is only $7.5 \mathrm{nT}$. This demonstrates that the results of the two methods are actually very similar.

Figure 14 shows a probability density function (pdf) of the disturbance field $B_{x}$ (i.e. the $B_{x}$ field after subtraction of the baseline) according to both methods. For comparison, a third baseline method is also included in this graph: this is the "traditional" baseline method, using the average value of the quietest day of each month as a baseline for the entire month (marked as "QD aver").

Also this graph demonstrates the similarity in the results of the baseline method from this paper and the SuperMAG baseline: the shapes of the two distributions are very similar, 


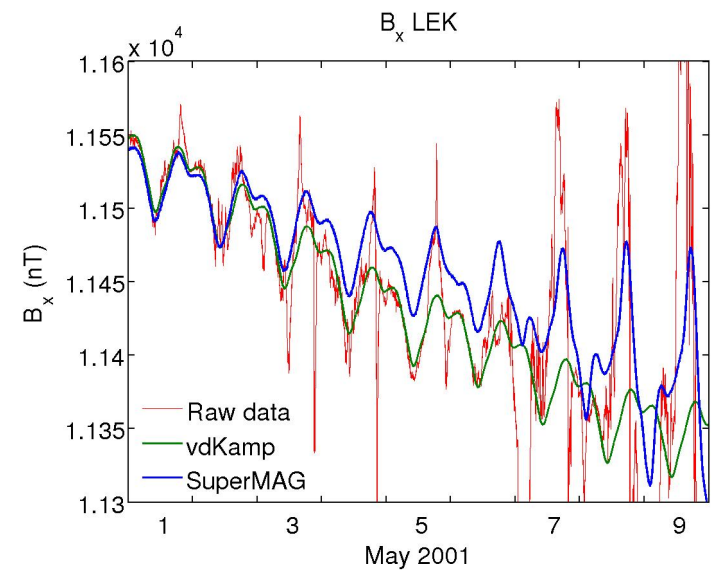

Fig. 15. $B_{x}$ field measured in Leknes on 1-9 May 2001 (red), the baseline from this paper (green), and the SuperMAG baseline (blue).

as well as the standard deviations (marked in the graph). A notable difference is however that the disturbance field according to SuperMAG shows a peak in the centre: it is equal to $0 \mathrm{nT}$ (within the $1 \mathrm{nT}$ resolution of the SuperMAG data) for a larger portion of time than would be expected from the shape of the rest of the distribution. Apparently, during this portion of time (10.3\% of this period of data), the SuperMAG baseline is exactly equal to the raw measured data. The same was noted for other periods of data.

The disturbance field using the "QD aver" baseline clearly shows a wider spread than using the other two methods (this can also be seen from its standard deviation). This effect is due to the $S_{q}$ variations, which occur every day, and using this baseline method are included in the resulting field. The effect of this on the pdf is that near-zero values of $B_{x}$ become spread out over a larger range.

A second comparison was made for a different period of data: the $B_{x}$ field at Leknes, during February-May 2001. This station exhibited during this time some strong equipment effects. In addition, the raw data contain a few gaps, together $2.9 \mathrm{~h}$ over these 4 months.

Figure 15 shows the raw data and the two baselines of $B_{x}$ at this station on 1-9 May 2001. During this time, this magnetometer showed some strong equipment drift. It can be seen in this figure that the baseline of this paper follows the drift better than the SuperMAG baseline, especially on 46 May. The result of this can also be seen in the pdf of the disturbance field according to the two methods over FebruaryMay 2001, shown in Fig. 16. The increased probability for values between -15 and $-44 \mathrm{nT}$ using the SuperMAG baseline is due to the effect seen in Fig. 15.

It should however be noted that cases like this are rare in the databases. The more significant difference between the two methods is their availability. In some other parts of these data (not shown here), the magnetometer showed

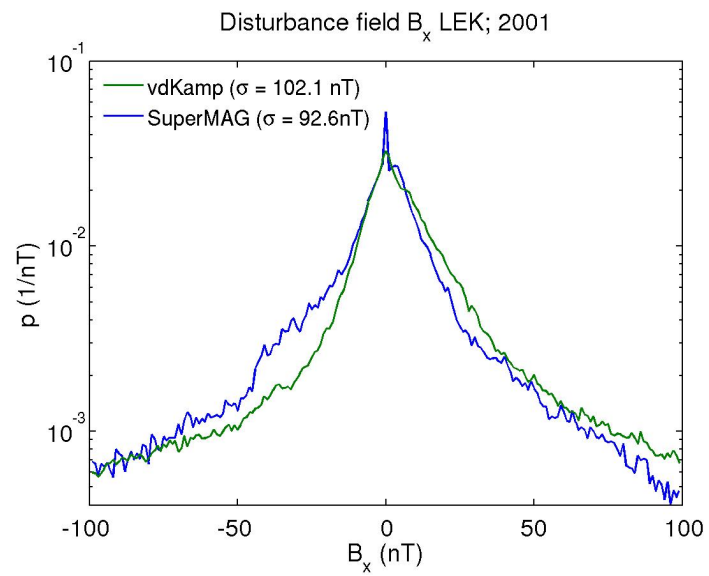

Fig. 16. Pdf of the $B_{x}$ disturbance field at Leknes over FebruaryMay 2001: using the baseline from this paper (availability $2877.1 \mathrm{~h}$ ), and the SuperMAG baseline (availability $2770.1 \mathrm{~h}$ ).

strong equipment effects which caused the data to be qualified as "invalid" in the SuperMAG database, and as a result $110 \mathrm{~h}$ of the data of $B_{x}$ in Leknes during February-May 2001 are unavailable there. However, the unavailability of the disturbance field using the baseline according to this paper is equal to that of the raw data in the IMAGE database over this period: $2.9 \mathrm{~h}$. The data patches with equipment effects are therefore included in the pdf of the disturbance field using the baseline of this paper, shown in Fig. 16. Still, this pdf shows a curve without any significant irregularities (and similar in shape to the one in Fig. 14), which demonstrates that these equipment effects were adequately removed from the data.

In addition, it can be noted that from many other magnetometers which showed equipment effects, such as Nordkapp and Sørøya, no or very few data are available from the SuperMAG website. Presumably, this is because these data were there classified as "invalid" due to the poor quality. (Note that the SuperMAG project concentrates more on the global magnetic field than on high spatial resolution, making the loss of a few measuring points not vital.)

It can be concluded from this comparison that the baseline according to this paper is in general not very different from that used in SuperMAG. It follows the day-to-day variations of the field slightly more. The main difference between the two is that the method from this paper is better able to deal with equipment effects without having to remove much data. This allows a higher spatial resolution in critical areas, such as the auroral oval boundary north of Scandinavia.

\subsection{Error estimate}

A reliable evaluation of the error of the baseline procedure of this paper is not possible, since there is no "correct" baseline known in any magnetic field measurement. However, an estimate of the worst-case error can be made. This should 
be expected in the case of strong equipment effects, such as the equipment drift which happened in December 2009 in Sørøya, shown in Figs. 2 and 10. This is one of the strongest and most sudden variations due to equipment drift found in the database.

In Fig. 10, the $B_{x}$ field is seen to decrease quite suddenly between 22 and 24 December, but because of the other irregularities, it is hard to say exactly when the change started and ended. The long-term baseline, because of the way it is set up, shows a drift from 22 December, 12:00 UT, to 24 December, 12:00 UT.

However, visual inspection of the graph seems to suggest that the equipment drift might also have started at 23 December, 00:00 UT, and reached its end value on 24 December, 00:00 UT. As a test case, this development was used for an alternative long-term baseline. A full baseline based on this alternative long-term baseline led to a difference with the baseline according to this paper with a maximum of $27 \mathrm{nT}$ (which maximum occurred at 23 December, 00:00 UT), and being above $15 \mathrm{nT}$ for $16 \mathrm{~h}$. Hence, if the trace described above of the equipment drift is assumed correct, then that is the error in the baseline of this paper caused by this most extreme case of equipment drift. Note that other baseline procedures which do not take equipment drift into account would probably encounter due to this drift a much larger error, for a longer time: ignoring this equipment effect completely would lead to an error with a maximum of about $80 \mathrm{nT}$, and which would be above $15 \mathrm{nT}$ for about 18 days. (However, in most other projects, this section of data would probably be labelled as "invalid" because of the equipment drift.)

Since naturally occurring $S_{q}$ variations are much smaller than this equipment drift, and occur much less suddenly, the error caused by those should be expected to be much smaller than the values mentioned above. This is why the error estimate above can be seen as a worst-case error, with probably only a single occurrence in the entire database.

\section{Other applications}

Now that the harmonic coefficients $X_{\mathrm{h}}$ (e.g. in Eq. 1) and $Y_{\mathrm{h}}$ of the magnetometer baselines have been derived over $10 \mathrm{yr}$ and for 32 magnetometer stations, these can be used to examine their long-term behaviour, which represent the solar quiet-time $\left(S_{q}\right)$ diurnal variation of the magnetic field $B_{x}$ and $B_{y}$. Like the disturbance field, also this field is mainly caused by currents in the ionosphere. However, unlike the disturbance field, this field varies with diurnal variations of electron density in the ionosphere, which in turn depends on solar radiation.

Several statistical studies of the harmonic coefficients of $S_{q}$ diurnal variations, dependent on season, year, location and solar activity, have already been made throughout the 20th century, revealing information about the long-term dependencies of the geomagnetic field (e.g. see the overviews by Matsushita (1968) and Campbell (1989)). The results obtained in the study of the current paper can help to verify those studies, and help to improve prediction models of the $S_{q}$ magnetic field. This will be the subject of a later paper.

\section{Conclusions}

A novel method of determining the baselines of magnetometer data has been presented, which makes magnetometer data suitable for the calculation of ionospheric equivalent currents. The full baseline is composed of three components:

- a jump baseline, which contains only the artificial jumps in the data and is otherwise constant.

- a long-term baseline, linearly interpolated between daily median values of the raw data. The daily median values are calculated for all days except the most disturbed ones.

- a diurnal baseline, linearly interpolated between "templates", derived from the quiet days. These templates consist of the first seven harmonics of the diurnal variation of the magnetic field on the quiet days. This method ensures a smooth baseline at all times, avoiding any discontinuities at transitions between days or months.

The baseline derived according to this method is able to follow medium- to long-term variations in the measured magnetic field, such as tidal and secular variations, as well as equipment drifts of individual instruments. The method avoids many problems associated with traditional baseline methods and some of the other recently published methods, and is simpler in procedure than most other recent ones.

The resulting disturbance field (i.e. field after removal of the baseline) has a fairly flat frequency spectrum up to $f=1 /(4 \mathrm{~h})$, and decreases after that.

The baseline follows the day-to-day variations of the magnetic field slightly more than the SuperMAG baseline; the resulting disturbance field distribution is quite similar between these two baselines.

The main advantage of the baseline method of this paper over others is that it removes equipment drift and other artefacts efficiently without discarding too much data, so that even low-quality data from remote unmanned magnetometers can be made suitable. This can give valuable contributions to the equivalent current database, especially in the area near the polar cap boundary.

Acknowledgements. The research leading to these results has received funding from the European Community's Seventh Framework Programme (FP7/2007-2013) within the call for "Exploitation of Space Science and Exploration Data", under grant agreement no. 263325 (ECLAT project). 
For the IMAGE ground magnetometer data, the author would like to thank E. Tanskanen and the institutes that maintain the IMAGE magnetometer network.

For the SuperMAG baseline data, the author would like to thank J. Gjerloev, the Johns Hopkins Applied Physics Laboratory and the University of Bergen, Norway.

Edited by: M. Rose

\section{References}

Amm, O. and Viljanen, A.: Ionospheric disturbance magnetic field continuation from the ground to the ionosphere using spherical elementary current systems, Earth Planets Space, 51, 431-440, 1999.

Boteler, D. H., Pirjola, R. J., and Nevanlinna, H.: The effects of geomagnetic disturbances on electrical systems at the earth's surface, Adv. Space Res., 22, 17-27, 1998.

Campbell, W. H.: An Introduction to Quiet Daily Geomagnetic Fields, Pure Appl. Geophys., 131, 315-331, 1989.

Chapman, S. and Bartels, J.: Geomagnetism, Clarendon Press, Oxford, UK, 1940.

Davis, T. N. and Sugiura, M.: Auroral electrojet activity index AE and its universal time variations, J. Geophys. Res., 71, 785-801, 1966.

Gjerloev, J. W.: The SuperMAG data processing technique, J. Geophys. Res., 117, A09213, doi:10.1029/2012JA017683, 2012.

Janzhura, A. S. and Troshichev, O. A.: Determination of the running quiet daily geomagnetic variation, J. Atmos. Sol.-Terr. Phy., 70, 962-972, doi:10.1016/j.jastp.2007.11.004, 2008.

Kamide, Y. and Baumjohann, W.: Magnetosphere-Ionosphere Coupling, Physics and chemistry in space planetology, vol. 23, Springer-Verlag, New York, 178 pp., 1993.

Laakso, H., Perry, C., McCaffrey, S., Herment, D., Allen, A. J., Harvey, C. C., Escoubet, C. P., Gruenberger, C., Taylor, M. G. G. T., and Turner, R.: Cluster Active Archive: Overview, in: The Cluster Active Archive, edited by: Laakso, H., Taylor, M. G. T. T., and Escoubet, C. P., Astrophysics and Space Science Proceedings, Springer, Dordrecht, Heidelberg, London, New York, doi:10.1007/978-90-481-3499-1_1, 2009.
Matsushita, S.: Sq and L Current Systems in the Ionosphere, Geophys. J. Astr. Soc., 15, 109-125, 1968.

Pedatella, N. M., Forbes, J. M., and Richmond:, A. D.: Seasonal and longitudinal variations of the solar quiet $(\mathrm{Sq})$ current system during solar minimum determined by CHAMP satellite magnetic field observations, J. Geophys. Res., 116, A04317, doi:10.1029/2010JA016289, 2011.

Price, A. T. and Wilkins, G. A.: New methods for the analysis of geomagnetic fields and their application to the Sq field of 1932 33, Philos. T. Roy. Soc. A, 156, 31-98, 1963.

Pulkkinen, A., Amm, O., Viljanen, A., and BEAR Working Group: Ionospheric equivalent current distributions determined with the method of spherical elementary current systems, J. Geophys. Res., 108, 1053, doi:10.1029/2001JA005085, 2003.

Stauning, P.: Determination of the quiet daily geomagnetic variations for polar regions, J. Atmos. Sol.-Terr. Phy., 73, 2314-2330, doi:10.1016/j.jastp.2011.07.004, 2011.

Sugiura, M.: Hourly values of equatorial Dst for the IGY, Annals of the International Geophysical Year, Vol. XXXV, Part I, International Council of Scientific Unions, Pergamon Press, Oxford, London, Edinburgh, New York, Paris, Frankfurt, 9-45, 1964.

Untiedt, J. and Baumjohann, W.: Studies of polar current systems using the IMS Scandinavian magnetometer array, Space Sci. Rev., 63, 245-390, 1993.

Viljanen, A. and Häkkinen, L.: IMAGE magnetometer network, in: Satellite-Ground Based Coordination Sourcebook, edited by: Lockwood, M., Wild, M. N., and Opgenoorth, H. J., ESA publications SP-1198, ESTEC, Noordwijk, the Netherlands, 111-117, 1997.

Viljanen, A., Nevanlinna, H., Pajunpää, K., and Pulkkinen, A.: Time derivative of the horizontal geomagnetic field as an activity indicator, Ann. Geophys., 19, 1107-1118, doi:10.5194/angeo-191107-2001, 2001. 